\title{
Sharp bounds for Neuman means in terms of two-parameter contraharmonic and arithmetic mean
}

Wei-Mao Qian' ${ }^{1}$ Zai-Yin $\mathrm{He}^{2}$, Hong-Wei Zhang ${ }^{3}$ and Yu-Ming Chu ${ }^{4 *}$ (D)

\section{"Correspondence:}

chuyuming2005@126.com

${ }^{4}$ Department of Mathematics,

Huzhou University, Huzhou, China

Full list of author information is

available at the end of the article

\section{Springer}

$$
\begin{aligned}
& \text { Abstract } \\
& \text { In the article, we prove that } \lambda_{1}=1 / 2+\sqrt{[(\sqrt{2}+\log (1+\sqrt{2})) / 2]^{1 / v}-1 / 2}, \\
& \mu_{1}=1 / 2+\sqrt{6 v} /(12 v), \lambda_{2}=1 / 2+\sqrt{[(\pi+2) / 4]^{1 / v}-1} / 2 \text { and } \mu_{2}=1 / 2+\sqrt{3} \\
& \text { the best possible parameters on the interval }[1 / 2,1] \text { such that the double } \\
& C^{v}\left[\lambda_{1} x+\left(1-\lambda_{1}\right) y, \lambda_{1} y+\left(1-\lambda_{1}\right) x\right] A^{1-v}(x, y) \\
& \quad<\mathcal{R}_{Q A}(x, y)<C^{v}\left[\mu_{1} x+\left(1-\mu_{1}\right) y, \mu_{1} y+\left(1-\mu_{1}\right) x\right] A^{1-v}(x, y), \\
& C^{v}\left[\lambda_{2} x+\left(1-\lambda_{2}\right) y, \lambda_{2} y+\left(1-\lambda_{2}\right) x\right] A^{1-v}(x, y) \\
& \quad<\mathcal{R}_{A Q}(x, y)<C^{v}\left[\mu_{2} x+\left(1-\mu_{2}\right) y, \mu_{2} y+\left(1-\mu_{2}\right) x\right] A^{1-v}(x, y)
\end{aligned}
$$
$\mu_{1}=1 / 2+\sqrt{6 v} /(12 v), \lambda_{2}=1 / 2+\sqrt{[(\pi+2) / 4]^{1 / v}-1} / 2$ and $\mu_{2}=1 / 2+\sqrt{3 v} /(6 v)$ are the best possible parameters on the interval $[1 / 2,1]$ such that the double inequalities

hold for all $x, y>0$ with $x \neq y$ and $v \in[1 / 2, \infty)$, where $A(x, y)$ is the arithmetic mean, $C(x, y)$ is the contraharmonic mean, and $\mathcal{R}_{Q A}(x, y)$ and $\mathcal{R}_{A Q}(x, y)$ are two Neuman means.

MSC: $26 \mathrm{E} 60$

Keywords: Arithmetic mean; Quadratic mean; Contraharmonic mean; Schwab-Borchardt mean; Neuman mean; Two-parameter contraharmonic and arithmetic mean

\section{Introduction}

Let $x, y>0$. Then the arithmetic mean $A(x, y)$, quadratic mean $Q(x, y)$ [1], contraharmonic mean $C(x, y)[2,3]$, and Schwab-Borchardt mean $\mathrm{SB}(x, y)[4]$ are given by

$$
\begin{aligned}
& A(x, y)=\frac{x+y}{2}, \quad Q(x, y)=\sqrt{\frac{x^{2}+y^{2}}{2}}, \quad C(x, y)=\frac{x^{2}+y^{2}}{x+y}, \\
& \operatorname{SB}(x, y)= \begin{cases}\frac{\sqrt{y^{2}-x^{2}}}{\arccos (x / y)}, & x<y, \\
x, & x=y, \\
\frac{\sqrt{x^{2}-y^{2}}}{\cosh ^{-1}(x / y)}, & x>y,\end{cases}
\end{aligned}
$$

respectively, where $\cosh ^{-1}(\sigma)=\log \left(\sigma+\sqrt{\sigma^{2}-1}\right)$ is the inverse hyperbolic cosine function. 
The Gaussian arithmetic-geometric mean $\operatorname{AGM}(x, y)[5-7]$ of two positive real numbers $x$ and $y$ is defined by the common limit of the sequences $\left\{x_{n}\right\}_{n=0}^{\infty}$ and $\left\{y_{n}\right\}_{n=0}^{\infty}$, which are given by

$$
x_{0}=x, \quad y_{0}=y, \quad x_{n+1}=\frac{x_{n}+y_{n}}{2}, \quad y_{n+1}=\sqrt{x_{n} y_{n}} .
$$

It is well known that the bivariate means have wide applications in mathematics, physics, engineering, and other natural sciences [8-55], many special functions can be expressed using bivariate means, for example, the complete elliptic integral

$$
\mathcal{K}(r)=\int_{0}^{\pi / 2} \frac{d t}{\sqrt{1-r^{2} \sin ^{2}(t)}} \quad(0<r<1)
$$

of the first kind [56-61] and the modulus $\mu(r)$ of the plane Grötzsch ring [62,63] can be expressed by the Gaussian arithmetic-geometric mean $\operatorname{AGM}(x, y)$, the formula of the perimeter of an ellipse and the complete elliptic integral

$$
\mathcal{E}(r)=\int_{0}^{\pi / 2} \sqrt{1-r^{2} \sin ^{2}(t)} d t
$$

of the second kind [64-70] can be given in terms of the Toader mean [71-74]

$$
T(a, b)=\frac{2}{\pi} \int_{0}^{\pi / 2} \sqrt{a^{2} \cos ^{2}(t)+b^{2} \sin ^{2}(t)} d t .
$$

Indeed, we have

$$
\begin{aligned}
& \mathcal{K}(r)=\frac{\pi}{2} \frac{1}{\operatorname{AGM}\left(1, \sqrt{1-r^{2}}\right)}, \quad \mu(r)=\frac{\pi}{2} \frac{\operatorname{AGM}\left(1, \sqrt{1-r^{2}}\right)}{\operatorname{AGM}(1, r)}, \\
& L(x, y)=2 \pi T(x, y), \quad \mathcal{E}(r)=\frac{\pi}{2} T\left(1, \sqrt{1-r^{2}}\right) .
\end{aligned}
$$

Recently, the inequalities for bivariate means have attracted the attention of many mathematicians. Neuman [75] introduced the Neuman means

$$
\begin{aligned}
& \mathcal{R}_{Q A}(x, y)=\frac{1}{2}\left[Q(x, y)+\frac{A^{2}(x, y)}{\mathrm{SB}(Q(x, y), A(x, y))}\right], \\
& \mathcal{R}_{A Q}(x, y)=\frac{1}{2}\left[A(x, y)+\frac{Q^{2}(x, y)}{\mathrm{SB}(A(x, y), Q(x, y))}\right]
\end{aligned}
$$

and provided the formulas

$$
\begin{aligned}
& \mathcal{R}_{Q A}(x, y)=\frac{1}{2} A(x, y)\left[\sqrt{1+u^{2}}+\frac{\sinh ^{-1}(u)}{u}\right], \\
& \mathcal{R}_{A Q}(x, y)=\frac{1}{2} A(x, y)\left[1+\frac{\left(1+u^{2}\right) \arctan (u)}{u}\right]
\end{aligned}
$$


if $x>y>0$, where $u=(x-y) /(x+y)$ and $\sinh ^{-1}(\sigma)=\log \left(\sigma+\sqrt{\sigma^{2}+1}\right)$ is the inverse hyperbolic sine function. Neuman [4] proved that the inequalities

$$
A(x, y)<\mathcal{R}_{Q A}(x, y)<\mathcal{R}_{A Q}(x, y)<Q(x, y)
$$

hold for $x, y>0$ with $x \neq y$.

Zhang et al. [76] proved that $\alpha_{1}=1 / 2+\sqrt{2 \sqrt{2} \log (1+\sqrt{2})+\log ^{2}(1+\sqrt{2})-2} / 4=$ $0.7817 \ldots, \beta_{1}=1 / 2+\sqrt{3} / 6=0.7886 \ldots, \alpha_{2}=1 / 2+\sqrt{\pi^{2}+4 \pi-12} / 8=0.9038 \ldots$ and $\beta_{2}=1 / 2+\sqrt{6} / 6=0.9082 \ldots$ are the best possible parameters on the interval $[1 / 2,1]$ such that the double inequalities

$$
\begin{aligned}
& Q\left[\alpha_{1} x+\left(1-\alpha_{1}\right) y, \alpha_{1} y+\left(1-\alpha_{1}\right) x\right] \\
& \quad<\mathcal{R}_{Q A}(x, y)<Q\left[\beta_{1} x+\left(1-\beta_{1}\right) y, \beta_{1} y+\left(1-\beta_{1}\right) x\right], \\
& Q\left[\alpha_{2} x+\left(1-\alpha_{2}\right) y, \alpha_{2} y+\left(1-\alpha_{2}\right) x\right] \\
& \quad<\mathcal{R}_{A Q}(x, y)<Q\left[\beta_{2} x+\left(1-\beta_{2}\right) y, \beta_{2} y+\left(1-\beta_{2}\right) x\right]
\end{aligned}
$$

hold for $x, y>0$ with $x \neq y$.

In [77], Yang et al. proved that the double inequalities

$$
\begin{aligned}
\alpha & {\left[\frac{C(x, y)}{3}+\frac{2 A(x, y)}{3}\right]+(1-\alpha) C^{1 / 3}(x, y) A^{2 / 3}(x, y) } \\
& <\mathcal{R}_{A Q}(x, y)<\beta\left[\frac{C(x, y)}{3}+\frac{2 A(x, y)}{3}\right]+(1-\beta) C^{1 / 3}(x, y) A^{2 / 3}(x, y), \\
\lambda & {\left[\frac{C(x, y)}{6}+\frac{5 A(x, y)}{6}\right]+(1-\lambda) C^{1 / 6}(x, y) A^{5 / 6}(x, y) } \\
& <\mathcal{R}_{Q A}(x, y)<\mu\left[\frac{C(x, y)}{6}+\frac{5 A(x, y)}{6}\right]+(1-\mu) C^{1 / 6}(x, y) A^{5 / 6}(x, y)
\end{aligned}
$$

hold for for $x, y>0$ with $x \neq y$ if and only if $\alpha \leq(3 \pi+6-12 \sqrt[3]{2}) /(16-12 \sqrt[3]{2})=0.3470 \ldots$, $\beta \geq 2 / 5, \lambda \leq[3 \sqrt{2}+3 \log (1+\sqrt{2})-6 \sqrt[6]{2}] /(7-6 \sqrt[6]{2})=0.5730 \ldots$ and $\mu \geq 16 / 25$.

The main purpose of the article is to generalize inequalities (1.5) and (1.6). To achieve this goal, we define the two-parameter contraharmonic and arithmetic mean $W_{\lambda, v}(x, y)$ as follows:

$$
W_{\lambda, v}(x, y)=C^{v}[\lambda x+(1-\lambda) y, \lambda y+(1-\lambda) x] A^{1-v}(x, y),
$$

where $\lambda \in[1 / 2,1]$ and $v \in[1 / 2, \infty)$. We clearly see that the function $\lambda \rightarrow W_{\lambda, v}(x, y)$ is strictly increasing on $[1 / 2,1]$ for $v \in[1 / 2, \infty)$ and $x, y>0$ with $x \neq y$.

It follows from (1.1), (1.4) and (1.7) that

$$
\begin{aligned}
& W_{\lambda, 1 / 2}(x, y)=Q[\lambda x+(1-\lambda) y, \lambda y+(1-\lambda) x], \\
& W_{\lambda, 1}(x, y)=C[\lambda x+(1-\lambda) y, \lambda y+(1-\lambda) x], \\
& W_{1 / 2, v}(x, y)=A(x, y),
\end{aligned}
$$




$$
\begin{aligned}
& W_{1, v}(x, y)=C^{v}(x, y) A^{1-v}(x, y)=A(x, y)\left[\frac{Q(x, y)}{A(x, y)}\right]^{2 v} \geq Q(x, y), \\
& W_{1 / 2, v}(x, y)<\mathcal{R}_{Q A}(x, y)<\mathcal{R}_{A Q}(x, y)<W_{1, v}(x, y) .
\end{aligned}
$$

Inequalities (1.5), (1.6), and (1.10) give us the motivation to discuss the question: What are the best possible parameters $\lambda_{1}=\lambda_{1}(v), \mu_{1}=\mu_{1}(v), \lambda_{2}=\lambda_{2}(v)$ and $\mu_{2}=\mu_{2}(v)$ on the interval $[1 / 2,1]$ such that the double inequalities

$$
\begin{aligned}
& W_{\lambda_{1}, v}(x, y)<\mathcal{R}_{Q A}(x, y)<W_{\mu_{1}, v}(x, y), \\
& W_{\lambda_{2}, v}(x, y)<\mathcal{R}_{A Q}(x, y)<W_{\mu_{2}, v}(x, y)
\end{aligned}
$$

hold for all $x, y>0$ with $x \neq y$ and $v \in[1 / 2, \infty)$ ?

\section{Lemmas}

In order to prove our main results, we need to introduce and establish five lemmas which we present in this section.

Lemma 2.1 ([78, Theorem 1.25]) Let $\alpha, \beta \in \mathbb{R}$ with $\alpha<\beta, \Gamma, \Psi:[\alpha, \beta] \rightarrow \mathbb{R}$ be continuous on $[\alpha, \beta]$ and differentiable on $(\alpha, \beta)$ with $\Psi^{\prime}(\tau) \neq 0$ on $(\alpha, \beta)$. Then the functions

$$
\frac{\Gamma(\tau)-\Gamma(\alpha)}{\Psi(\tau)-\Psi(\alpha)}, \quad \frac{\Gamma(\tau)-\Gamma(\beta)}{\Psi(\tau)-\Psi(\beta)}
$$

are (strictly) increasing (decreasing) on $(\alpha, \beta)$ if $\Gamma^{\prime}(\tau) / \Psi^{\prime}(\tau)$ is (strictly) increasing (decreasing) on $(\alpha, \beta)$.

\section{Lemma 2.2 The function}

$$
\phi(t)=\frac{\sqrt{1+t^{2}} \sinh ^{-1}(t)}{t}
$$

is strictly increasing from $(0,1)$ onto $(1, \sqrt{2} \log (1+\sqrt{2}))$.

Proof Differentiating $\phi(t)$ gives

$$
\phi^{\prime}(t)=\frac{\phi_{1}(t)}{t \sqrt{1+t^{2}}},
$$

where

$$
\phi_{1}(t)=t \sqrt{1+t^{2}}-\sinh ^{-1}(t)
$$

It follows from (2.2) that

$$
\begin{aligned}
& \phi_{1}\left(0^{+}\right)=0, \\
& \phi_{1}^{\prime}(t)=\frac{2 t^{2}}{\sqrt{1+t^{2}}}>0
\end{aligned}
$$

for all $t \in(0,1)$. 
Note that

$$
\phi\left(0^{+}\right)=1, \quad \phi\left(1^{-}\right)=\sqrt{2} \log (1+\sqrt{2}) .
$$

Therefore, Lemma 2.2 follows from (2.1) and (2.3)-(2.5).

\section{Lemma 2.3 The function}

$$
\varphi(t)=\frac{t^{3}}{\left(1+t^{2}\right) \arctan (t)-t}
$$

is strictly increasing from $(0,1)$ onto $(3 / 2,2 /(\pi-2))$.

Proof Let $\varphi_{1}(t)=t^{3}$ and $\varphi_{2}(t)=\left(1+t^{2}\right) \arctan (t)-t$. Then we clearly see that

$$
\begin{aligned}
& \varphi_{1}\left(0^{+}\right)=\varphi_{2}\left(0^{+}\right), \quad \varphi(t)=\frac{\varphi_{1}(t)}{\varphi_{2}(t)}, \\
& \frac{\varphi_{1}^{\prime}(t)}{\varphi_{2}^{\prime}(t)}=\frac{3 t}{2 \arctan (t)} .
\end{aligned}
$$

It is not difficult to verify that the function $t \mapsto t / \arctan (t)$ is strictly increasing from $(0,1)$ onto $(1,4 / \pi)$. Then equation (2.7) leads to the conclusion that $\varphi_{1}^{\prime}(t) / \varphi_{2}^{\prime}(t)$ is strictly increasing on $(0,1)$.

Note that

$$
\varphi\left(0^{+}\right)=\frac{3}{2}, \quad \varphi\left(1^{-}\right)=\frac{2}{\pi-2} .
$$

Therefore, Lemma 2.3 follows from Lemma 2.1, (2.6), (2.8), and the monotonicity of $\varphi_{1}^{\prime}(t) / \varphi_{2}^{\prime}(t)$.

Lemma 2.4 Let $\theta \in[0,1], v \in[1 / 2, \infty), t \in(0,1)$ and

$$
f_{\theta, v}(t)=v \log \left(1+\theta t^{2}\right)-\log \left[t \sqrt{1+t^{2}}+\sinh ^{-1}(t)\right]+\log t+\log 2
$$

Then we have the following two conclusions:

(1) $f_{\theta, v}(t)>0$ for all $t \in(0,1)$ if and only if $\theta \geq 1 /(6 v)$;

(2) $f_{\theta, v}(t)<0$ for all $t \in(0,1)$ if and only if $\theta \leq[(\sqrt{2}+\log (1+\sqrt{2})) / 2]^{1 / v}-1$.

Proof It follows from (2.9) that

$$
\begin{aligned}
& f_{\theta, v}\left(0^{+}\right)=0, \\
& f_{\theta, v}\left(1^{-}\right)=v \log (1+\theta)-\log [\sqrt{2}+\log (1+\sqrt{2})]+\log 2, \\
& f_{\theta, v}^{\prime}(t)=\frac{t\left[(2 v-1)\left(t \sqrt{1+t^{2}}-\sinh ^{-1}(t)\right)+4 v \sinh ^{-1}(t)\right]}{\left(1+\theta t^{2}\right)\left[t \sqrt{1+t^{2}}+\sinh ^{-1}(t)\right]}\left[\theta-f_{v}(t)\right],
\end{aligned}
$$

where

$$
f_{v}(t)=\frac{t \sqrt{1+t^{2}}-\sinh ^{-1}(t)}{(2 v-1) t^{2}\left[t \sqrt{1+t^{2}}-\sinh ^{-1}(t)\right]+4 v t^{2} \sinh ^{-1}(t)} .
$$


Let $\psi_{1}(t)=t \sqrt{1+t^{2}}-\sinh ^{-1}(t)$ and $\psi_{2}(t)=(2 v-1) t^{2}\left[t \sqrt{1+t^{2}}-\sinh ^{-1}(t)\right]+4 v t^{2} \sinh ^{-1}(t)$. Then

$$
\begin{aligned}
& \psi_{1}\left(0^{+}\right)=\psi_{2}\left(0^{+}\right)=0, \quad f_{v}(t)=\frac{\psi_{1}(t)}{\psi_{2}(t)}, \\
& \frac{\psi_{1}^{\prime}(t)}{\psi_{2}^{\prime}(t)}=\frac{1}{(2 v+1) \phi(t)+2(2 v-1) t^{2}+4 v-1},
\end{aligned}
$$

where $\phi(t)$ is defined in Lemma 2.2 .

Equation (2.14) and Lemma 2.2 imply that $\psi_{1}^{\prime}(t) / \psi_{2}^{\prime}(t)$ is strictly decreasing on $(0,1)$. Therefore, the conclusion that $f_{v}(t)$ is strictly decreasing on $(0,1)$ follows from Lemma 2.1 and (2.13), together with the monotonicity of $\psi_{1}^{\prime}(t) / \psi_{2}^{\prime}(t)$ on the interval $(0,1)$. Moreover, making use of L'Hôpital's rule, we have that

$$
\begin{aligned}
& f_{v}\left(0^{+}\right)=\frac{1}{6 v}, \\
& f_{v}\left(1^{-}\right)=\frac{\sqrt{2}-\log (1+\sqrt{2})}{(2 v-1) \sqrt{2}+(2 v+1) \log (1+\sqrt{2})}=: \theta_{0} .
\end{aligned}
$$

We divide the proof into three cases.

Case 1. $\theta \geq 1 /(6 v)$. Then (2.12) and (2.15), together with the monotonicity of $f_{v}(t)$ on the interval $(0,1)$, lead to the conclusion that $f_{\theta, v}(t)$ is strictly increasing on $(0,1)$. Therefore, $f_{\theta, v}(t)>0$ for all $t \in(0,1)$ follows from $(2.10)$ and the monotonicity of $f_{\theta, v}(t)$ on the interval $(0,1)$.

Case 2. $\theta \leq \theta_{0}$. Then from (2.12) and (2.16), together with the monotonicity of $f_{v}(t)$ on the interval $(0,1)$, we clearly see that $f_{\theta, v}(t)$ is strictly decreasing on $(0,1)$. Therefore, $f_{\theta, v}(t)<0$ for all $t \in(0,1)$ follows from $(2.10)$ and the monotonicity of $f_{\theta, v}(t)$ on the interval $(0,1)$.

Case 3. $\theta_{0}<\theta<1 /(6 v)$. Then from (2.12), (2.15), (2.16), and the monotonicity of $f_{v}(t)$ on the interval $(0,1)$, we clearly see that there exists $t_{0} \in(0,1)$ such that $f_{\theta, v}(t)$ is strictly decreasing on $\left(0, t_{0}\right)$ and strictly increasing on $\left(t_{0}, 1\right)$.

We divide the proof into two subcases.

Subcase 3.1. $[(\sqrt{2}+\log (1+\sqrt{2})) / 2]^{1 / v}-1<\theta<1 /(6 v)$. Then (2.11) leads to

$$
f_{\theta, v}\left(1^{-}\right)>0
$$

Therefore, there exists $t^{*} \in\left(t_{0}, 1\right)$ such that $f_{\theta, v}(t)<0$ for $t \in\left(0, t^{*}\right)$ and $f_{\theta, v}(t)>0$ for $t \in\left(t^{*}, 1\right)$ follows from $(2.10)$ and $(2.17)$, together with the piecewise monotonicity of $f_{\theta, v}(t)$ on the interval $(0,1)$.

Subcase 3.2. $\theta_{0}<\theta \leq[(\sqrt{2}+\log (1+\sqrt{2})) / 2]^{1 / v}-1$. Then $(2.11)$ leads to

$$
f_{\theta, v}\left(1^{-}\right) \leq 0
$$

Therefore, $f_{\theta, v}(t)<0$ for all $t \in(0,1)$ follows from $(2.10)$ and (2.18), together with the piecewise monotonicity of $f_{\theta, v}(t)$ on the interval $(0,1)$.

Lemma 2.5 Let $\vartheta \in[0,1], v \in[1 / 2, \infty), t \in(0,1)$ and

$$
g_{\vartheta, v}(t)=v \log \left(1+\vartheta t^{2}\right)-\log \left[t+\left(1+t^{2}\right) \arctan (t)\right]+\log (t)+\log 2 .
$$


Then the following statements are true:

(1) $g_{\vartheta, v}(t)>0$ for all $t \in(0,1)$ if and only if $\vartheta \geq 1 /(3 v)$;

(2) $g_{\vartheta, v}(t)<0$ for all $t \in(0,1)$ if and only if $\vartheta \leq[(\pi+2) / 4]^{1 / v}-1$.

Proof It follows from (2.19) that

$$
\begin{aligned}
& g_{\vartheta, v}\left(0^{+}\right)=0, \\
& g_{\vartheta, v}\left(1^{-}\right)=v \log (1+\vartheta)-\log \left(\frac{\pi+2}{4}\right), \\
& g_{\vartheta, v}^{\prime}(t)=\frac{t\left[\left((2 v-1) t^{2}+2 v+1\right) \arctan (t)+(2 v-1) t\right]}{\left(1+\vartheta t^{2}\right)\left[t+\left(1+t^{2}\right) \arctan (t)\right]}\left[\vartheta-g_{\nu}(t)\right],
\end{aligned}
$$

where

$$
g_{v}(t)=\frac{t-\left(1-t^{2}\right) \arctan (t)}{t^{2}\left[\left((2 v-1) t^{2}+2 v+1\right) \arctan (t)+(2 v-1) t\right]} .
$$

Let $\omega_{1}(t)=\left[t-\left(1-t^{2}\right) \arctan (t)\right] / t^{2}$ and $\omega_{2}(t)=\left[(2 v-1) t^{2}+2 v+1\right] \arctan (t)+(2 v-1) t$. Then elaborate computations lead to

$$
\begin{aligned}
& \omega_{1}\left(0^{+}\right)=\omega_{2}\left(0^{+}\right)=0, \quad g_{v}(t)=\frac{\omega_{1}(t)}{\omega_{2}(t)}, \\
& \frac{\omega_{1}^{\prime}(t)}{\omega_{2}^{\prime}(t)}=\frac{1}{2\left[(2 v-1) t^{2}+v\right] \varphi(t)+(2 v-1) t^{4}},
\end{aligned}
$$

where $\varphi(t)$ is defined in Lemma 2.3.

From Lemma 2.3 and $(2.24)$ we know that $\omega_{1}^{\prime}(t) / \omega_{2}^{\prime}(t)$ is strictly decreasing on $(0,1)$. Therefore, the conclusion that $g_{v}(t)$ is strictly decreasing on $(0,1)$ follows from Lemma 2.1 and (2.23), together with the monotonicity of $\omega_{1}^{\prime}(t) / \omega_{2}^{\prime}(t)$ on the interval $(0,1)$. Moreover, making use of L'Hôpital's rule, we have that

$$
\begin{aligned}
& g_{v}\left(0^{+}\right)=\frac{1}{3 v}, \\
& g_{v}\left(1^{-}\right)=\frac{1}{(\pi+2) v-1} .
\end{aligned}
$$

We divide the proof into three cases.

Case $1 . \vartheta \geq 1 /(3 v)$. Then (2.22) and (2.25), together with the monotonicity of $g_{v}(t)$ on the interval $(0,1)$, lead to the conclusion that $g_{\vartheta, v}(t)$ is strictly increasing on $(0,1)$. Therefore, $g_{\vartheta, v}(t)>0$ for all $t \in(0,1)$ follows from $(2.20)$ and the monotonicity of $g_{\vartheta, v}(t)$ on the interval $(0,1)$.

Case 2. $\vartheta \leq 1 /[(\pi+2) v-1]$. Then from (2.22) and (2.26), together with the monotonicity of $g_{v}(t)$ on the interval $(0,1)$, we clearly see that $g_{\vartheta, v}(t)$ is strictly decreasing on $(0,1)$. Therefore, $g_{\vartheta, \nu}(t)<0$ for all $t \in(0,1)$ follows from $(2.20)$ and the monotonicity of $g_{\vartheta, \nu}(t)$ on the interval $(0,1)$.

Case 3. $1 /[(\pi+2) v-1]<\vartheta<1 /(6 v)$. Then it follows from (2.22), (2.25), (2.26), and the monotonicity of $g_{\nu}(t)$ on the interval $(0,1)$ that there exists $\rho_{0} \in(0,1)$ such that $g_{\vartheta, v}(t)$ is strictly decreasing on $\left(0, \rho_{0}\right)$ and strictly increasing on $\left(\rho_{0}, 1\right)$. 
We divide the proof into two subcases.

Subcase 3.1. $[(\pi+2) / 4]^{1 / v}-1<\vartheta<1 /(6 v)$. Then $(2.21)$ leads to

$$
g_{\vartheta, v}\left(1^{-}\right)>0 \text {. }
$$

Therefore, there exists $\rho^{*} \in\left(\rho_{0}, 1\right)$ such that $g_{\vartheta, v}(t)<0$ for $t \in\left(0, \rho^{*}\right)$ and $g_{\vartheta, v}(t)>0$ for $t \in$ $\left(\rho^{*}, 1\right)$ follows from $(2.20)$ and $(2.27)$, together with the piecewise of $g_{\vartheta, v}(t)$ on the interval $(0,1)$.

Subcase 3.2. $1 /[(\pi+2) v-1]<\vartheta \leq[(\pi+2) / 4]^{1 / v}-1$. Then $(2.21)$ gives

$$
g_{\vartheta, v}\left(1^{-}\right) \leq 0
$$

Therefore, $g_{\vartheta, v}(t)<0$ for all $t \in(0,1)$ follows from $(2.20)$ and (2.28), together with the piecewise of $g_{\vartheta, v}(t)$ on the interval $(0,1)$.

\section{Main results}

Theorem 3.1 Let $\lambda_{1}, \mu_{1} \in[1 / 2,1]$ and $v \in[1 / 2, \infty)$. Then the double inequality

$$
W_{\lambda_{1}, v}(x, y)<\mathcal{R}_{Q A}(x, y)<W_{\mu_{1}, v}(x, y)
$$

holds for all $x, y>0$ with $x \neq y$ if and only if $\lambda_{1} \leq 1 / 2+\sqrt{[(\sqrt{2}+\log (1+\sqrt{2})) / 2]^{1 / v}-1} / 2$ and $\mu_{1} \geq 1 / 2+\sqrt{6 v} /(12 v)$.

Proof Since both $W_{\theta, v}(x, y)$ and $\mathcal{R}_{Q A}(x, y)$ are symmetric and homogenous of degree 1 , without loss of generality, we assume that $x>y>0$. Let $t=(x-y) /(x+y) \in(0,1)$ and $\theta \in[1 / 2,1]$. Then from (1.1), (1.2), and (1.7) we get

$$
\begin{aligned}
& \frac{\left.W_{\theta, v}(x, y)\right)}{A(x, y)}=\left[1+(2 \theta-1)^{2} t^{2}\right]^{v}, \\
& \frac{\mathcal{R}_{Q A}(x, y)}{A(x, y)}=\frac{1}{2}\left[\sqrt{1+t^{2}}+\frac{\sinh ^{-1}(t)}{t}\right] .
\end{aligned}
$$

It follows from (3.2) and (3.3) that

$$
\begin{aligned}
\log \left[\frac{W_{\theta, v}(x, y)}{\mathcal{R}_{Q A}(x, y)}\right]= & \log \left[\frac{W_{\theta, v}(x, y)}{A(x, y)}\right]-\log \left[\frac{\mathcal{R}_{Q A}(x, y)}{A(x, y)}\right] \\
= & v \log \left[1+(2 \theta-1)^{2} t^{2}\right]-\log \left[t \sqrt{1+t^{2}}+\sinh ^{-1}(t)\right] \\
& +\log (t)+\log 2 .
\end{aligned}
$$

Therefore, Theorem 3.1 follows easily from Lemma 2.4 and (3.4).

Theorem 3.2 Let $\lambda_{2}, \mu_{2} \in[1 / 2,1]$ and $v \in[1 / 2, \infty)$. Then the double inequality

$$
W_{\lambda_{2}, v}(x, y)<\mathcal{R}_{A Q}(x, y)<W_{\mu_{2}, v}(x, y)
$$

holds for all $x, y>0$ with $x \neq y$ if and only if $\lambda_{2} \leq 1 / 2+\sqrt{[(\pi+2) / 4]^{1 / v}-1} / 2$ and $\mu_{2} \geq$ $1 / 2+\sqrt{3 v} /(6 v)$. 
Proof Since both $W_{\vartheta, v}(x, y)$ and $\mathcal{R}_{A Q}(x, y)$ are symmetric and homogenous of degree 1 , without loss of generality, we assume that $x>y>0$. Let $t=(x-y) /(x+y) \in(0,1)$ and $\vartheta \in[1 / 2,1]$. Then it follows from (1.1), (1.3), and (1.7) that

$$
\begin{aligned}
& \frac{\left.W_{\vartheta, v}(x, y)\right)}{A(x, y)}=\left[1+(2 \vartheta-1)^{2} t^{2}\right]^{\nu}, \\
& \frac{\mathcal{R}_{A Q}(x, y)}{A(x, y)}=\frac{1}{2}\left[1+\frac{\left(1+t^{2}\right) \arctan (t)}{t}\right] .
\end{aligned}
$$

From (3.6) and (3.7) we have

$$
\begin{aligned}
\log \left[\frac{\left.W_{\vartheta, v}(x, y)\right)}{\mathcal{R}_{A Q}(x, y)}\right]= & \log \left[\frac{W_{\vartheta, v}(x, y)}{A(x, y)}\right]-\log \left[\frac{\mathcal{R}_{A Q}(x, y)}{A(x, y)}\right] \\
= & v \log \left[1+(2 \vartheta-1)^{2} t^{2}\right]-\log \left[t+\left(1+t^{2}\right) \arctan (t)\right] \\
& +\log (t)+\log 2
\end{aligned}
$$

Therefore, Theorem 3.2 follows easily from Lemma 2.5 and (3.8).

Remark 3.3 Let $v=1 / 2$. Then from (1.8) we clearly see that Theorems 3.1 and 3.2 become (1.5) and (1.6), respectively.

Let $v=1$. Then from (1.9) and Theorems 3.1 and 3.2 we get Corollary 3.4 immediately.

Corollary 3.4 Let $\lambda_{1}, \mu_{1}, \lambda_{2}, \mu_{2} \in[1 / 2,1]$. Then the double inequalities

$$
\begin{aligned}
& C\left[\lambda_{1} x+\left(1-\lambda_{1}\right) y, \lambda_{1} y+\left(1-\lambda_{1}\right) x\right]<\mathcal{R}_{Q A}(x, y)<C\left[\mu_{1} x+\left(1-\mu_{1}\right) y, \mu_{1} y+\left(1-\mu_{1}\right) x\right], \\
& C\left[\lambda_{2} x+\left(1-\lambda_{2}\right) y, \lambda_{2} y+\left(1-\lambda_{2}\right) x\right]<\mathcal{R}_{A Q}(x, y)<C\left[\mu_{2} x+\left(1-\mu_{2}\right) y, \mu_{2} y+\left(1-\mu_{2}\right) x\right]
\end{aligned}
$$

hold for all $x, y>0$ with $x \neq y$ if and only if $\lambda_{1} \leq 1 / 2+\sqrt{[(\sqrt{2}+\log (1+\sqrt{2})) / 2]-1} / 2=$ $0.6922 \ldots, \mu_{1} \geq 1 / 2+\sqrt{6} / 12=0.7041 \ldots, \lambda_{2} \leq 1 / 2+\sqrt{[(\pi+2) / 4]-1} / 2=0.7671 \ldots$ and $\mu_{2} \geq 1 / 2+\sqrt{3} / 6=0.7886 \ldots$.

Let $u \in(0,1), x=1+u, y=1-u, \lambda_{1}=1 / 2+\sqrt{[(\sqrt{2}+\log (1+\sqrt{2})) / 2]^{1 / v}-1} / 2, \mu_{1}=1 / 2+$ $\sqrt{6 v} /(12 v), \lambda_{2}=1 / 2+\sqrt{[(\pi+2) / 4]^{1 / v}-1} / 2$ and $\mu_{2}=1 / 2+\sqrt{3 v} /(6 v)$. Then (1.2), (1.3), and Theorems 3.1 and 3.2 lead to Corollary 3.5.

Corollary 3.5 The double inequalities

$$
\begin{aligned}
& 2\left[\left(1-u^{2}\right)+\left(\frac{\sqrt{2}+\log (1+\sqrt{2})}{2}\right)^{1 / v} u^{2}\right]^{v}-\sqrt{1+u^{2}} \\
& <\frac{\sinh ^{-1}(u)}{u}<2\left(1+\frac{u^{2}}{6 v}\right)^{v}-\sqrt{1+u^{2}}, \\
& \frac{2\left[\left(1-u^{2}\right)+\left(\frac{2+\pi}{4}\right)^{1 / v} u^{2}\right]^{v}-1}{1+u^{2}}<\frac{\arctan (u)}{u}<\frac{2\left(1+\frac{1}{3 v} u^{2}\right)^{v}-1}{1+u^{2}}
\end{aligned}
$$

hold for all $u \in(0,1)$ and $v \in[1 / 2, \infty)$. 


\section{Results and discussion}

In the article, we give the sharp bounds for the Neuman means

$$
\mathcal{R}_{Q A}(x, y)=\frac{1}{2}\left[Q(x, y)+\frac{A^{2}(x, y)}{\mathrm{SB}(Q(x, y), A(x, y))}\right]
$$

and

$$
\mathcal{R}_{A Q}(x, y)=\frac{1}{2}\left[A(x, y)+\frac{Q^{2}(x, y)}{\mathrm{SB}(A(x, y), Q(x, y))}\right]
$$

in terms of the two-parameter contraharmonic and arithmetic mean

$$
W_{\lambda, v}(x, y)=C^{\nu}[\lambda x+(1-\lambda) y, \lambda y+(1-\lambda) x] A^{1-v}(x, y),
$$

and find new bounds for the functions $\sinh (u) / u$ and $\arctan (u) / u$ on the interval $(0,1)$.

\section{Conclusion}

In the article, we prove that the double inequalities

$$
W_{\lambda_{1}, v}(x, y)<\mathcal{R}_{Q A}(x, y)<W_{\mu_{1}, v}(x, y), \quad W_{\lambda_{2}, v}(x, y)<\mathcal{R}_{A Q}(x, y)<W_{\mu_{2}, v}(x, y)
$$

hold for all $x, y>0$ with $x \neq y$ if and only if $\lambda_{1} \leq 1 / 2+\sqrt{[(\sqrt{2}+\log (1+\sqrt{2})) / 2]^{1 / v}-1} / 2$, $\mu_{1} \geq 1 / 2+\sqrt{6 v} /(12 v), \lambda_{2} \leq 1 / 2+\sqrt{[(\pi+2) / 4]^{1 / v}-1} / 2$ and $\mu_{2} \geq 1 / 2+\sqrt{3 v} /(6 v)$ if $\lambda_{1}, \mu_{1}, \lambda_{2}, \mu_{2} \in[1 / 2,1]$ and $v \in[1 / 2, \infty)$. Our results are a natural generalization of some previously known results, and our approach may lead to many follow-up studies.

\section{Acknowledgements}

The authors would like to express their sincere thanks to the editor and the anonymous reviewers for their helpful comments and suggestions.

\section{Funding}

The work was supported by the Natural Science Foundation of China (Grant Nos. 61673169, 11301127, 11701176, $11626101,11601485)$ and the Natural Science Foundation of Huzhou City (Grant No. 2018YZ07).

Availability of data and materials

Not applicable.

\section{Competing interests}

The authors declare that they have no competing interests.

\section{Authors' contributions}

All authors contributed equally to the writing of this paper. All authors read and approved the final manuscript.

\section{Author details}

${ }^{1}$ School of Continuing Education, Huzhou Vocational \& Technical College, Huzhou, China. ${ }^{2}$ College of Mathematics and Econometrics, Hunan University, Changsha, China. ${ }^{3}$ School of Mathematics and Statistics, Changsha University of Science \& Technology, Changsha, China. ${ }^{4}$ Department of Mathematics, Huzhou University, Huzhou, China.

\section{Publisher's Note}

Springer Nature remains neutral with regard to jurisdictional claims in published maps and institutional affiliations. 


\section{References}

1. Chu, H.-H., Qian, W.-M., Chu, Y.-M., Song, Y.-Q.: Optimal bounds for a Toader-type mean in terms of one-parameter quadratic and contraharmonic means. J. Nonlinear Sci. Appl. 9(5), 3424-3432 (2016)

2. Chu, Y.-M., Hou, S.-W.: Sharp bounds for Seiffert mean in terms of contraharmonic mean. Abstr. Appl. Anal. 2012, Article ID 425175 (2012)

3. Chu, Y.-M., Wang, M.-K., Ma, X.-Y.: Sharp bounds for Toader mean in terms of contraharmonic mean with applications. J. Math. Inequal. 7(2), 161-166 (2013)

4. Neuman, E., Sándor, J.: On the Schwab-Borchardt mean. Math. Pannon. 14(2), 253-266 (2003)

5. Chu, Y.-M., Wang, M.-K.: Inequalities between arithmetic-geometric, Gini, and Toader means. Abstr. Appl. Anal. 2012, Article ID 830585 (2012)

6. Qian, W.-M., Chu, Y.-M.: Sharp bounds for a special quasi-arithmetic mean in terms of arithmetic and geometric means with two parameters. J. Inequal. Appl. 2017, Article ID 274 (2017)

7. Yang, Z.-H., Qian, W.-M., Chu, Y.-M., Zhang, W.: On approximating the arithmetic-geometric mean and complete elliptic integral of the first kind. J. Math. Anal. Appl. 462(2), 1714-1726 (2018)

8. Lin, L., Liu, Z.-Y.: An alternating projected gradient algorithm for nonnegative matrix factorization. Appl. Math. Comput. 217(24), 9997-10002 (2011)

9. Liu, Z.-Y., Santos, J., Ralha, R.: On computing complex square roots of real matrices. Appl. Math. Lett. 25(10), $1565-1568(2012)$

10. Jiang, Y.-J., Ma, J.-T.: Spectral collocation methods for Volterra-integro differential equations with noncompact kernels J. Comput. Appl. Math. 244, 115-124 (2013)

11. Li, X.-F., Tang, G.-J., Tang, B.-Q.: Stress field around a strike-slip fault in orthotropic elastic layers via a hypersingular integral equation. Comput. Math. Appl. 66(11), 2317-2326 (2013)

12. Qin, G.-X., Huang, C.-X., Xie, Y.-Q., Wen, F.-H.: Asymptotic behavior for third-order quasi-linear differential equations. Adv. Differ. Equ. 2013, Article ID 305 (2013)

13. Tang, W.-S., Sun, Y.-J.: Construction of Runge-Kutta type methods for solving ordinary differential equations. Appl. Math. Comput. 234, 179-191 (2014)

14. Huang, C.-X., Yang, Z.-C., Yi, T.-S., Zou, X.-F.: On the basins of attraction for a class of delay differential equations with non-monotone bistable nonlinearities. J. Differ. Equ. 256(7), 2101-2114 (2014)

15. Huang, C.-X., Guo, S., Liu, L.-Z.: Boundedness on Morrey space for Toeplitz type operator associated to singular integral operator with variable Calderón-Zygmund kernel. J. Math. Inequal. 8(3), 453-464 (2014)

16. Xie, D.-Q., Li, J.: A new analysis of electrostatic free energy minimization and Poisson-Boltzmann equation for protein in ionic solvent. Nonlinear Anal., Real World Appl. 21, 185-196 (2015)

17. Dai, Z.-F., Chen, X.-H., Wen, F.-H.: A modified Perry's conjugate gradient method-based derivative-free method for solving large-scale nonlinear monotone equations. Appl. Math. Comput. 270, 378-386 (2015)

18. Wang, W.-S.: High order stable Runge-Kutta methods for nonlinear generalized pantograph equations on the geometric mesh. Appl. Math. Model. 39(1), 270-283 (2015)

19. Tang, Y.-X., Jing, K.: Existence and global exponential stability of almost periodic solution for delayed competitive neural networks with discontinuous activations. Math. Methods Appl. Sci. 39(11), 2821-2839 (2016)

20. Li, J.-L., Sun, G.-Y., Zhang, R.-M.: The numerical solution of scattering by infinite rough interfaces based on the integral equation method. Comput. Math. Appl. 71(7), 1491-1502 (2016)

21. Dai, Z.-F.: Comments on a new class of nonlinear conjugate gradient coefficients with global convergence properties. Appl. Math. Comput. 276, 297-300 (2016)

22. Yang, Z.-H., Qian, W.-M., Chu, Y.-M., Zhang, W.: On rational bounds for the gamma function. J. Inequal. Appl. 2017, Article ID 210 (2017)

23. Adil Khan, M., Chu, Y.-M., Khan, T.U., Khan, J.: Some new inequalities of Hermite-Hadamard type for s-convex functions with applications. Open Math. 15(1), 1414-1430 (2017)

24. Li, J., Liu, F.-W., Feng, L.-B., Turner, I.W.: A novel finite volume method for the Riesz space distributed-order advection-diffusion equation. Appl. Math. Model. 46, 536-553 (2017)

25. Liu, Z.-Y., Qin, X.-R., Wu, N.-C., Zhang, Y.-L.: The shifted classical circulant and skew circulant splitting iterative methods for Toeplitz matrices. Can. Math. Bull. 60(4), 807-815 (2017)

26. Duan, L., Huang, L.-H., Guo, Z.-Y., Fang, X.-W.: Periodic attractor for reaction-diffusion higher-order Hopfield neural networks with time-varying delays. Comput. Math. Appl. 73(2), 233-245 (2017)

27. Yang, C., Huang, L.-H.: New criteria on exponential synchronization and existence of periodic solutions of complex BAM networks with delays. J. Nonlinear Sci. Appl. 10(10), 5464-5482 (2017)

28. Duan, L., Huang, C.-X.: Existence and global attractivity of almost periodic solutions for a delayed differential neoclassical growth model. Math. Methods Appl. Sci. 40(3), 814-822 (2017)

29. Huang, C.-X., Liu, L.-Z.: Boundedness of multilinear singular integral operator with a non-smooth kernel and mean oscillation. Quaest. Math. 40(3), 295-312 (2017)

30. Xi, H.-Y., Huang, L.-H., Qiao, Y.-C., Li, H.-Y., Huang, C.-X.: Permanence and partial extinction in a delayed three-species food chain model with stage structure and time-varying coefficients. J. Nonlinear Sci. Appl. 10(12), 6177-6191 (2017)

31. Huang, T.-R., Han, B.-W., Ma, X.-Y., Chu, Y.-M.: Optimal bounds for the generalized Euler-Mascheroni constant. J. Inequal. Appl. 2018, Article ID 118 (2018)

32. Adil Khan, M., Chu, Y.-M., Kashuri, A., Liko, R., Ali, G.: Conformable fractional integrals versions of Hermite-Hadamard inequalities and their generalizations. J. Funct. Spaces 2018, Article ID 6928130 (2018)

33. Adil Khan, M., Khurshid, Y., Du, T.-S., Chu, Y.-M.: Generalization of Hermite-Hadamard type inequalities via conformable fractional integrals. J. Funct. Spaces 2018, Article ID 5357463 (2018)

34. Zhao, T.-H., Wang, M.-K., Zhang, W., Chu, Y.-M.: Quadratic transformation inequalities for Gaussian hypergeometric function. J. Inequal. Appl. 2018, Article ID 251 (2018)

35. Tang, W.-S., Zhang, J.-J.: Symplecticity-preserving continuous-stage Runge-Kutta-Nyström methods. Appl. Math. Comput. 233, 204-219 (2018)

36. Liu, Z.-Y., Wu, N.-C., Qin, X.-R., Zhang, Y.-L.: Trigonometric transform splitting methods for real symmetric Toeplitz systems. Comput. Math. Appl. 75(8), 2782-2794 (2018) 
37. Zhu, K.-X., Xie, Y.-Q., Zhou, F.: Pullback attractors for a damped semilinear wave equation with delays. Acta Math. Sin. 34(7), 1131-1150 (2018)

38. Zhang, Y.: On products of consecutive arithmetic progressions II. Acta Math. Hung. 156(1), 240-254 (2018)

39. Cai, Z.-W., Huang, J.-H., Huang, L.-H.: Periodic orbit analysis for the delayed Filippov system. Proc. Am. Math. Soc. 146(11), 4667-4682 (2018)

40. Adil Khan, M., Begum, S., Khurshid, Y., Chu, Y.-M.: Ostrowski type inequalities involving conformable fractional integrals. J. Inequal. Appl. 2018, Article ID 70 (2018)

41. Huang, C.-X., Qiao, Y.-C., Huang, L.-H., Agarwal, R.P.: Dynamical behaviors of a food-chain model with stage structure and time delays. Adv. Differ. Equ. 2018, Article ID 186 (2018)

42. Duan, L., Fang, X.-W., Huang, C.-X.: Global exponential convergence in a delayed almost periodic Nicholson's blowflies model with discontinuous harvesting. Math. Methods Appl. Sci. 41(5), 1954-1965 (2018)

43. Tan, Y.-X., Huang, C.-X., Sun, B., Wang, T.: Dynamics of a class of delayed reaction-diffusion system with Neumann boundary condition. J. Math. Anal. Appl. 458(2), 1115-1130 (2018)

44. Qiu, S.-L., Ma, X.-Y., Chu, Y.-M.: Sharp Landen transformation inequalities for hypergeometric functions, with applications. J. Math. Anal. Appl. 474(2), 1306-1337 (2019)

45. Wang, M.-K., Chu, Y.-M., Zhang, W.: Monotonicity and inequalities involving zero-balanced hypergeometric function. Math. Inequal. Appl. 22(2), 601-617 (2019)

46. Li, J., Ying, J.-Y., Xie, D.-X.: On the analysis and applications of an ion size-modified Poisson-Boltzmann equation. Nonlinear Anal., Real World Appl. 47, 188-203 (2019)

47. Li, Y., Li, J., Wen, P.H.: Finite and infinite block Petrov-Galerkin method for cracks in functionally graded materials. Appl. Math. Model. 68, 306-326 (2019)

48. Jiang, Y.-J., Xu, X.-J.: A monotone finite volume methods for time fractional Fokker-Planck equations. Sci. China Math. 62(4), 783-794 (2019)

49. Peng, J., Zhang, Y.: Heron triangles with figurate numbers sides. Acta Math. Hung. 157(2), 478-488 (2019)

50. Wang, J.-F., Chen, X.-Y., Huang, L.-H.: The number and stability of limit cycles for planar piecewise linear systems of node-saddle type. J. Math. Anal. Appl. 469(1), 405-427 (2019)

51. Wang, J.-F., Huang, C.-X., Huang, L.-H.: Discontinuity-induced limit cycles in a general planar piecewise linear system of saddle-focus type. Nonlinear Anal. Hybrid Syst. 33, 162-178 (2019)

52. Zhao, T.-H., Zhou, B.-C., Wang, M.-K., Chu, Y.-M.: On approximating the quasi-arithmetic mean. J. Inequal. Appl. 2019 Article ID 42 (2019)

53. Khurshid, Y., Adil Khan, M., Chu, Y.-M.: Conformable integral inequalities of the Hermite-Hadamard type in terms of GG- and GA-convexities. J. Funct. Spaces 2019, Article ID 6926107 (2019)

54. Khurshid, Y., Adil Khan, M., Chu, Y.-M., Khan, Z.A.: Hermite-Hadamard-Fejér inequalities for conformable fractional integrals via preinvex functions. J. Funct. Spaces 2019, Article ID 3146210 (2019)

55. Zaheer Ullah, S., Adil Khan, M., Khan, Z.A., Chu, Y.-M.: Integral majorization type inequalities for the function in the sense of strong convexity. J. Funct. Spaces 2019, Article ID 9487823 (2019)

56. Wang, M.-K., Chu, Y.-M., Qiu, Y.-F., Qiu, S.-L.: An optimal power mean inequality for the complete elliptic integrals. Appl. Math. Lett. 24(6), 887-890 (2011)

57. Chu, Y.-M., Wang, M.-K., Qiu, Y.-F.: On Alzer and Qiu's conjecture for complete elliptic integral and inverse hyperbolic tangent function. Abstr. Appl. Anal. 2011, Article ID 697547 (2011)

58. Wang, M.-K., Chu, Y.-M., Qiu, S.-L., Jiang, Y.-P.: Convexity of the complete elliptic integrals of the first kind with respect to Hölder means. J. Math. Anal. Appl. 388(2), 1141-1146 (2012)

59. Chu, Y.-M., Qiu, Y.-F., Wang, M.-K.: Hölder mean inequalities for the complete elliptic integrals. Integral Transforms Spec. Funct. 23(7), 521-527 (2012)

60. Wang, G.-D., Zhang, X.-H., Chu, Y.-M.: A power mean inequality involving the complete elliptic integrals. Rocky Mt. J. Math. 44(5), 1661-1667 (2014)

61. Yang, Z.-H., Qian, W.-M., Chu, Y.-M.: Monotonicity properties and bounds involving the complete elliptic integrals of the first kind. Math. Inequal. Appl. 21(4), 1185-1199 (2018)

62. Wang, G.-D., Zhang, X.-H., Chu, Y.-M.: A power mean inequality for the Grötzsch ring function. Math. Inequal. Appl. 14(4), 833-837 (2011)

63. Qiu, S.-L., Qiu, Y.-F., Wang, M.-K., Chu, Y.-M.: Hölder mean inequalities for the generalized Grötzsch ring and Hersch-Pfluger distortion functions. Math. Inequal. Appl. 15(1), 237-245 (2012)

64. Wang, M.-K., Qiu, S.-L., Chu, Y.-M., Jiang, Y.-P.: Generalized Hersch-Pfluger distortion function and complete elliptic integrals. J. Math. Anal. Appl. 385(1), 221-229 (2012)

65. Chu, Y.-M., Wang, M.-K., Qiu, S.-L., Jiang, Y.-P.: Bounds for complete elliptic integrals of the second kind with applications. Comput. Math. Appl. 63(7), 1177-1184 (2012)

66. Chu, Y.-M., Wang, M.-K., Jiang, Y.-P., Qiu, S.-L.: Concavity of the complete elliptic integrals of the second kind with respect to Hölder means. J. Math. Anal. Appl. 395(2), 637-642 (2012)

67. Wang, M.-K., Chu, Y.-M.: Asymptotical bounds for complete elliptic integrals of the second kind. J. Math. Anal. Appl. 402(1), 119-126 (2013)

68. Yang, Z.-H., Qian, W.-M., Chu, Y.-M., Zhang, W.: Monotonicity rule for the quotient of two functions and its application. J. Inequal. Appl. 2017, Article ID 106 (2017)

69. Huang, T.-R., Tan, S.-Y., Ma, X.-Y., Chu, Y.-M.: Monotonicity properties and bounds for the complete $p$-elliptic integrals. J. Inequal. Appl. 2018, Article ID 239 (2018)

70. Yang, Z.-H., Chu, Y.-M., Zhang, W.: High accuracy asymptotic bounds for the complete elliptic integral of the second kind. Appl. Math. Comput. 348, 552-564 (2019)

71. Chu, Y.-M., Wang, M.-K., Qiu, S.-L., Qiu, Y.-F.: Sharp generalized Seiffert mean bounds for Toader mean. Abstr. Appl. Anal. 2011, Article ID 605259 (2011)

72. Chu, Y.-M., Wang, M.-K., Qiu, S.-L.: Optimal combinations bounds of root-square and arithmetic means for Toader mean. Proc. Indian Acad. Sci. Math. Sci. 122(1), 41-51 (2012)

73. Chu, Y.-M., Wang, M.-K.: Optimal Lehmer mean bounds for the Toader mean. Results Math. 61(3-4), 223-229 (2012)

74. Wang, J.-L., Qian, W.-M., He, Z.-Y., Chu, Y.-M.: On approximating the Toader mean by other bivariate means. J. Funct. Spaces 2019, Article ID 6082413 (2019) 
75. Neuman, E.: On a new bivariate mean. Aequ. Math. 88(3), 277-289 (2014)

76. Zhang, Y., Chu, Y.-M., Jiang, Y.-L.: Sharp geometric means bounds for Neuman means. Abstr. Appl. Anal. 2014, Article ID $949815(2014)$

77. Yang, Y.-Y., Qian, W.-M., Chu, Y.-M.: Refinements of bounds for Neuman means with applications. J. Nonlinear Sci. Appl. 9(4), 1529-1540 (2016)

78. Anderson, G.D., Vamanamurthy, M.K., Vuorinen, M.: Conformal Invariants, Inequalities, and Quasiconformal Maps. Wiley, New York (1997)

Submit your manuscript to a SpringerOpen ${ }^{\odot}$ journal and benefit from:

- Convenient online submission

$\checkmark$ Rigorous peer review

- Open access: articles freely available online

- High visibility within the field

- Retaining the copyright to your article

Submit your next manuscript at $\gg$ springeropen.com 